\title{
The Multiplicities of a Dual-thin $Q$-polynomial Association Scheme
}

\author{
Bruce E. Sagan \\ Department of Mathematics \\ Michigan State University \\ East Lansing, MI 44824-1027 \\ sagan@math.msu .edu \\ and \\ John S. Caughman, IV \\ Department of Mathematical Sciences \\ Portland State University \\ P. O. Box 751 \\ Portland, OR 97202-0751 \\ caughman@mth.pdx.edu
}

Submitted: June 23, 2000; Accepted: January 28, 2001.

MR Subject Classification: 05E30

\begin{abstract}
Let $Y=\left(X,\left\{R_{i}\right\}_{0 \leq i \leq D}\right)$ denote a symmetric association scheme, and assume that $Y$ is $Q$-polynomial with respect to an ordering $E_{0}, \ldots, E_{D}$ of the primitive idempotents. Bannai and Ito conjectured that the associated sequence of multiplicities $m_{i}(0 \leq i \leq D)$ of $Y$ is unimodal. Talking to Terwilliger, Stanton made the related conjecture that $m_{i} \leq m_{i+1}$ and $m_{i} \leq m_{D-i}$ for $i<D / 2$. We prove that if $Y$ is dual-thin in the sense of Terwilliger, then the Stanton conjecture is true.
\end{abstract}

\section{Introduction}

For a general introduction to association schemes, we refer to [1], [2], [5], or [9]. Our notation follows that found in [3].

Throughout this article, $Y=\left(X,\left\{R_{i}\right\}_{0 \leq i \leq D}\right)$ will denote a symmetric, $D$-class association scheme. Our point of departure is the following well-known result of Taylor and Levingston.

1.1 Theorem. [7] If $Y$ is $P$-polynomial with respect to an ordering $R_{0}, \ldots, R_{D}$ of the associate classes, then the corresponding sequence of valencies

$$
k_{0}, k_{1}, \ldots, k_{D}
$$


is unimodal. Furthermore,

$$
k_{i} \leq k_{i+1} \quad \text { and } \quad k_{i} \leq k_{D-i} \quad \text { for } i<D / 2 .
$$

Indeed, the sequence is log-concave, as is easily derived from the inequalities $b_{i-1} \geq b_{i}$ and $c_{i} \leq c_{i+1}(0<i<D)$, which are satisfied by the intersection numbers of any $P$-polynomial scheme (cf. [5, p. 199]).

In their book on association schemes, Bannai and Ito made the dual conjecture.

1.2 Conjecture. [1, p. 205] If $Y$ is $Q$-polynomial with respect to an ordering $E_{0}, \ldots, E_{D}$ of the primitive idempotents, then the corresponding sequence of multiplicities

$$
m_{0}, m_{1}, \ldots, m_{D}
$$

is unimodal.

Bannai and Ito further remark that although unimodality of the multiplicities follows easily whenever the dual intersection numbers satisfy the inequalities $b_{i-1}^{*} \geq b_{i}^{*}$ and $c_{i}^{*} \leq$ $c_{i+1}^{*}(0<i<D)$, unfortunately these inequalities do not always hold. For example, in the Johnson scheme $J\left(k^{2}, k\right)$ we find that $c_{k-1}^{*}>c_{k}^{*}$ whenever $k>3$.

Talking to Terwilliger, Stanton made the following related conjecture.

1.3 Conjecture. [8] If $Y$ is $Q$-polynomial with respect to an ordering $E_{0}, \ldots, E_{D}$ of the primitive idempotents, then the corresponding multiplicities satisfy

$$
m_{i} \leq m_{i+1} \quad \text { and } \quad m_{i} \leq m_{D-i} \quad \text { for } i<D / 2 .
$$

Our main result shows that under a suitable restriction on $Y$, these last inequalities are satisfied.

To state our result more precisely, we first review a few definitions. Let $\operatorname{Mat}_{X}(\mathbb{C})$ denote the $\mathbb{C}$-algebra of matrices with entries in $\mathbb{C}$, where the rows and columns are indexed by $X$, and let $A_{0}, \ldots, A_{D}$ denote the associate matrices for $Y$. Now fix any $x \in X$, and for each integer $i(0 \leq i \leq D)$, let $E_{i}^{*}=E_{i}^{*}(x)$ denote the diagonal matrix in $\operatorname{Mat}_{X}(\mathbb{C})$ with $y y$ entry

$$
\left(E_{i}^{*}\right)_{y y}=\left\{\begin{array}{ll}
1 & \text { if } x y \in R_{i}, \\
0 & \text { if } x y \notin R_{i} .
\end{array} \quad(y \in X) .\right.
$$

The Terwilliger algebra for $Y$ with respect to $x$ is the subalgebra $T=T(x)$ of $\operatorname{Mat}_{X}(\mathbb{C})$ generated by $A_{0}, \ldots, A_{D}$ and $E_{0}^{*}, \ldots, E_{D}^{*}$. The Terwilliger algebra was first introduced in [9] as an aid to the study of association schemes. For any $x \in X, T=T(x)$ is a finite dimensional, semisimple $\mathbb{C}$-algebra, and is noncommutative in general. We refer to [3] or [9] for more details. $T$ acts faithfully on the vector space $V:=\mathbb{C}^{X}$ by matrix multiplication. $V$ is endowed with the inner product $\langle$,$\rangle defined by \langle u, v\rangle:=u^{t} \bar{v}$ for all $u, v \in V$. Since $T$ is semisimple, $V$ decomposes into a direct sum of irreducible $T$-modules.

Let $W$ denote an irreducible $T$-module. Observe that $W=\sum E_{i}^{*} W$ (orthogonal direct sum), where the sum is taken over all the indices $i(0 \leq i \leq D)$ such that $E_{i}^{*} W \neq 0$. We set

$$
d:=\left|\left\{i: E_{i}^{*} W \neq 0\right\}\right|-1,
$$


and note that the dimension of $W$ is at least $d+1$. We refer to $d$ as the diameter of $W$. The module $W$ is said to be thin whenever $\operatorname{dim}\left(E_{i}^{*} W\right) \leq 1(0 \leq i \leq D)$. Note that $W$ is thin if and only if the diameter of $W$ equals $\operatorname{dim}(W)-1$. We say $Y$ is thin if every irreducible $T(x)$-module is thin for every $x \in X$.

Similarly, note that $W=\sum E_{i} W$ (orthogonal direct sum), where the sum is over all $i(0 \leq i \leq D)$ such that $E_{i} W \neq 0$. We define the dual diameter of $W$ to be

$$
d^{*}:=\left|\left\{i: E_{i} W \neq 0\right\}\right|-1,
$$

and note that $\operatorname{dim} W \geq d^{*}+1$. A dual thin module $W$ satisfies $\operatorname{dim}\left(E_{i} W\right) \leq 1(0 \leq i \leq D)$. So $W$ is dual thin if and only if $\operatorname{dim}(W)=d^{*}+1$. Finally, $Y$ is dual thin if every irreducible $T(x)$-module is dual thin for every vertex $x \in X$.

Many of the known examples of $Q$-polynomial schemes are dual thin. (See [10] for a list.) Our main theorem is as follows.

1.4 Theorem. Let $Y$ denote a symmetric association scheme which is $Q$-polynomial with respect to an ordering $E_{0}, \ldots, E_{D}$ of the primitive idempotents. If $Y$ is dual-thin, then the multiplicities satisfy

$$
m_{i} \leq m_{i+1} \quad \text { and } \quad m_{i} \leq m_{D-i} \quad \text { for } i<D / 2 .
$$

The proof of Theorem 1.4 is contained in the next section.

We remark that if $Y$ is bipartite $P$ - and $Q$-polynomial, then it must be dual-thin and $m_{i}=m_{D-i}$ for $i<D / 2$. So Theorem 1.4 implies the following corollary. (cf. [4, Theorem 9.6]).

1.5 Corollary. Let $Y$ denote a symmetric association scheme which is bipartite $P$ - and $Q$-polynomial with respect to an ordering $E_{0}, \ldots, E_{D}$ of the primitive idempotents. Then the corresponding sequence of multiplicities

$$
m_{0}, m_{1}, \ldots, m_{D}
$$

is unimodal.

1.6 Remark. By recent work of Ito, Tanabe, and Terwilliger [6], the Stanton inequalities (Conjecture 1.3) have been shown to hold for any $Q$-polynomial scheme which is also $P$ polynomial. In other words, our Theorem 1.4 remains true if the words "dual-thin" are replaced by "P-polynomial".

\section{Proof of the Theorem}

Let $Y=\left(X,\left\{R_{i}\right\}_{0 \leq i \leq D}\right)$ denote a symmetric association scheme which is $Q$-polynomial with respect to the ordering $E_{0}, \ldots, E_{D}$ of the primitive idempotents. Fix any $x \in X$ and let $T=T(x)$ denote the Terwilliger algebra for $Y$ with respect to $x$. Let $W$ denote any irreducible $T$-module. We define the dual endpoint of $W$ to be the integer $t$ given by

$$
t:=\min \left\{i: 0 \leq i \leq D, E_{i} W \neq 0\right\}
$$


We observe that $0 \leq t \leq D-d^{*}$, where $d^{*}$ denotes the dual diameter of $W$.

2.1 Lemma. [9, p.385] Let $Y$ be a symmetric association scheme which is $Q$-polynomial with respect to the ordering $E_{0}, \ldots, E_{D}$ of the primitive idempotents. Fix any $x \in X$, and write $E_{i}^{*}=E_{i}^{*}(x)(0 \leq i \leq D), T=T(x)$. Let $W$ denote an irreducible $T$-module with dual endpoint $t$. Then

(i) $E_{i} W \neq 0 \quad$ iff $\quad t \leq i \leq t+d^{*} \quad(0 \leq i \leq D)$.

(ii) Suppose $W$ is dual-thin. Then $W$ is thin, and $d=d^{*}$.

2.2 Lemma. [3, Lemma 4.1] Under the assumptions of the previous lemma, the dual endpoint $t$ and diameter $d$ of any irreducible $T$-module satisfy

$$
2 t+d \geq D
$$

Proof of Theorem 1.4. Fix any $x \in X$, and let $T=T(x)$ denote the Terwilliger algebra for $Y$ with respect to $x$. Since $T$ is semisimple, there exists a positive integer $s$ and irreducible $T$-modules $W_{1}, W_{2}, \ldots, W_{s}$ such that

$$
V=W_{1}+W_{2}+\cdots+W_{s} \quad \text { (orthogonal direct sum). }
$$

For each integer $j, 1 \leq j \leq s$, let $t_{j}$ (respectively, $d_{j}^{*}$ ) denote the dual endpoint (respectively, dual diameter) of $W_{j}$. Now fix any nonnegative integer $i<D / 2$. Then for any $j$, $1 \leq j \leq s$,

$$
\begin{aligned}
E_{i} W_{j} \neq 0 & \Rightarrow t_{j} \leq i & & (\text { by Lemma 2.1(i)) } \\
& \Rightarrow t_{j}<i+1 \leq D-i \leq D-t_{j} & & (\text { since } i<D / 2) \\
& \Rightarrow t_{j}<i+1 \leq D-i \leq t_{j}+d_{j}^{*} & & \text { (by Lemmas 2.1(ii), 2.2) } \\
& \Rightarrow E_{i+1} W_{j} \neq 0 \text { and } E_{D-i} W_{j} \neq 0 & & \text { (by Lemma 2.1(i)). }
\end{aligned}
$$

So we can now argue that, since $Y$ is dual thin,

$$
\begin{aligned}
\operatorname{dim}\left(E_{i} V\right) & =\left|\left\{j: 0 \leq j \leq s, E_{i} W_{j} \neq 0\right\}\right| \\
& \leq\left|\left\{j: ; 0 \leq j \leq s, E_{i+1} W_{j} \neq 0\right\}\right| \\
& =\operatorname{dim}\left(E_{i+1} V\right) .
\end{aligned}
$$

In other words, $m_{i} \leq m_{i+1}$. Similarly,

$$
\begin{aligned}
\operatorname{dim}\left(E_{i} V\right) & =\left|\left\{j: 0 \leq j \leq s, E_{i} W_{j} \neq 0\right\}\right| \\
& \leq\left|\left\{j: 0 \leq j \leq s, E_{D-i} W_{j} \neq 0\right\}\right| \\
& =\operatorname{dim}\left(E_{D-i} V\right)
\end{aligned}
$$

This yields $m_{i} \leq m_{D-i}$. 


\section{References}

[1] E. Bannai and T. Ito, "Algebraic Combinatorics I: Association Schemes," Benjamin/Cummings, London, 1984.

[2] A. E. Brouwer, A. M. Cohen, and A. Neumaier, "Distance-Regular Graphs," SpringerVerlag, Berlin, 1989.

[3] J. S. Caughman IV, The Terwilliger algebra for bipartite $P$ - and $Q$-polynomial association schemes, in preparation.

[4] J. S. Caughman IV, Spectra of bipartite $P$ - and $Q$-polynomial association schemes, Graphs Combin., to appear.

[5] C. D. Godsil, "Algebraic Combinatorics," Chapman and Hall, New York, 1993.

[6] T. Ito, K. Tanabe, and P. Terwilliger, Some algebra related to $P$ - and $Q$-polynomial association schemes, preprint.

[7] D. E. Taylor and R. Levingston, Distance-regular graphs, in "Combinatorial Mathematics, Proc. Canberra 1977," D. A. Holton and J. Seberry eds., Lecture Notes in Mathematics, Vol. 686, Springer-Verlag, Berlin, 1978, 313-323.

[8] P. Terwilliger, private communication.

[9] P. Terwilliger, The subconstituent algebra of an association scheme. I, J. Algebraic Combin. 1 (1992) 363-388.

[10] P. Terwilliger, The subconstituent algebra of an association scheme. III, J. Algebraic Combin. 2 (1993) 177-210. 